\section{Net profits}

C. E. Purdom and A. Preston of the Ministry of Agriculture, Fisheries and Food, Fisheries Laboratory, Lowestoft, write about the prospects for fish farming in the UK.

THE annual world production of 1 farmed fish is approximately $4 \times 10^{6}$ tons which represents about $15 \%$ of supplies of fish for direct human consumption. In the UK, production at present is about 1,500 tons a year, or less than $0.2 \%$ of consumption. Precise statistics are not available, but a considerable proportion, perhaps half, of the production is for conservation or sport purposes.

The fish farming industry in the UK comprises a variety of small, mostly privately owned, farms and a few large companies. About 100 private farms exist, each producing between 5 and 200 tons per year with by far the greater proportion at the low end of the scale; in addition, about 30 farms under the control of Regional Water Authorities produce fish for re-stocking purposes. Small scale fish farming has a history going back about 100 years. It is traditionally associated with the production of trout for sport purposes, although current expansion also includes food production. Involvement by large companies is very recent and is still in an early developmental phase, with production primarily for food supplies. Output is still low and geared to the supply of luxury food in the form of trout and salmon, with research emphasis on the latter. So far there is little input into the mass retail industry and marketing methods remain traditional.

Apart from a very minor effort with cyprinid fish for re-stocking, fish farming in the UK is at present restricted to salmonids. The culture in fresh water of native brown trout (Salmo trutta) and North American rainbow trout $(S$. gairdnerii) is the dominant element, with sea-water culture of salmon $(S$. salar) restricted to large companies with production levels of only a few hundred tons per year at present.

Marine fish farming research in the UK was initiated by the Ministry of Agriculture, Fisheries and Food (MAFF) about 15 years ago but its origins go back much further than this and probably to the turn of the century when hatcheries were established for plaice (Pleuronectes platessa) and cod (Gadus morhua) to produce fry for liberation into the sea for conservation purposes. This ineffective measure was discontinued by about 1920 , but the concept of farming marine fish seems to have grown out of the experience gained in the production and hatching of eggs of the plaice. Thus initial research was hatchery orientated and directed towards plaice farming. Hatchery techniques were successfully developed and large scale production of juvenile plaice became feasible by 1966 . At this time the White Fish Authority (WFA) entered the field to develop the rearing techniques towards commercially viable levels and, especially, to study the growing-on phase from juvenile stages to marketable size. It was in this area that economic problems with plaice became apparent; their food bill exceeded the first-sale value. Attention was therefore switched to sole (Solea solea) and turbot (Scophthalmus maximus) for which better economic returns might arise. Sole has proved more intractable than plaice during on-growing but turbot has been shown to be very amenable during this part of its life cycle. A basic requirement in intensive fish farming is that food is taken rapidly and not wasted or allowed to decompose and pollute the environment. Turbot fulfil this requirement but sole, with present rearing techniques, do not, being slow, cautious feeders. Progress in turbot farming has been restricted, however, by serious difficulties encountered in the production of juvenile fish and present research on farming techniques depends, in part, on the collection of juveniles from wild stocks. For conservation reasons this sets a restrictive limit on possible total commercial production for this species of about 100 tonnes a year. The earliest attempt to rear turbot was made by Anthony in 1910 but the first hatchery reared juveniles were not produced until 1972 and then only in very small numbers. Improvements have been made in succeeding years, during which the development of turbot hatchery techniques has had considerable priority in government laboratories, but the methods still fall short of the level of achievement necessary for commercial exploitation, although one major food company is exploring the hatchery production of turbot with the assistance of MAFF.

Fish farming in the UK has grown slowly in the freshwater and marine salmonid field under commercial patronage, although some acceleration has occurred over the past 2 or 3 years; on the other hand, government research, largely under the auspices of MAFF, but with the later involvement of the WFA and the Natural Environmental Research Council (NERC), has been preoccupied with the development of methods for rearing marine flatfish. So far no major research work on salmonid farming has been undertaken by government agencies, and commercial interest in marine flatfish has been limited, largely to a joint project between the WFA and one major food company. This dichotomy is in marked contrast to the situation in other parts of the world, such as North America, Japan and Norway, where government and private concerns are mutually and extensively involved in a wide variety of fish farming projects.

With little prospect of increasing the direct yield from the sea of species of prime fish, and many indications that the reverse might occur, it has become necessary to examine the potential for an expansion in fish farming in the UK. In this context a review was commissioned with the object of drawing up a MAFF programme of research and development into marine and freshwater fish cultivation and assessing the facilities required to carry it out. This review, which took into account the existing scope of fish farming activities, formed the view that a viable industry needed to be broadly based and to cater for both the small farmer and major commercial interests. Some legislative adjustments may be necessary in this context but the primary objective should be to provide, through a well conceived research and development programme, a sound scientific basis for the expansion of fish farming into a broadly based national industry.

The question of which species of fish to cultivate is crucial to the development of a research and development programme of relevance to an expanding industry. Among the salmonids, rainbow trout and Atlantic salmon are obvious choices in view of their present commercial status; brown trout and sea trout may also be of value within the context of marine culture of salmonids. Among the marine flatfish, turbot alone seems to have potential at this time, although sole would have definite economic advantages if the feeding problems experienced in the growingon phase could be solved. Other freshwater and marine species do not warrant attention at present but should be subject to periodic review in terms of their potential for cultivation.

The research and development needs differ between species. The principal subjects areas for investigation are hatchery studies, nutritional requirements, water management studies, facility development, genetic manipulation and disease control. Of these, hatchery techniques, nutrition and disease control are well established for salmonids after more than a century of development. There is still room for much improvement, particularly at the nutritional level, and disease control and treatment require continuous study. The major constraint to a large scale expansion in trout farming in fresh water, however, lies in the nature of the water requirements. Rainbow 
trout farms use about 30,000 gallons of high quality fresh water for each pound of fish produced and the availability of sites for expansion is very limited. Two methods for overcoming this constraint are the use of recycling systems and the culture of trout in sea water. The former can be regarded as a technological development based on present practices, whereas sea-water culture is a more radical change with many facets requiring investigation. Although no major research work on salmonid farming has been undertaken by MAFF or the Department of Agriculture and Fisheries for Scotland some facilities are available and plans for additional facilities are in hand: in these, special attention will be directed towards nutritional studies, cage systems in the sea, recirculation systems for fresh and salt water, and genetic studies aimed at producing improved strains. Disease research and monitoring is already covered both in England and Wales and in Scotland.

Flatfish farming research has not yet established a sufficiently reliable technology for full commercial development. The first priority for further work lies with turbot and especially with the need to overcome the problems of hatchery production of juveniles.

One major area of work of fundamental importance to any form of fish farming is the development of a genetic approach to produce improved strains, to avoid inbreeding depression and to conserve genetic uniformity and hence performance in domesticated lines of fish. A further useful field with genetic implications is the development of allyear-round spawning stocks and the control of sex itself. Genetic manipulation at the hatchery level is at present studied within the MAFF programme, but no facilities exist for extending this to the growing-on phase or for holding stocks of domesticated fish. For marine flatfish, the creation of domesticated lines has not yet begun. Similarly, Atlantic salmon stocks are basically of wild origin but rainbow trout exist in a wide variety of selected lines, particularly in North America. Although a proper evaluation of their merits, and the possibility of heterosis in F1 hybrids, seems not to have been attempted, this is an area of study which could have an immediate beneficial effect on farming practices in the UK.

In conclusion, there is an established and expanding industry for salmonid farming, principally in fresh water, and a growing interest on the part of large companies in marine flatfish culture. In view of the likely diminution of supplies of prime quality fish like sole and turbot from the sea it is desirable to encourage the continued expansion and development of this area of food production, on a broad basis and at all levels of commercial involvement, though this should not be construed as implying any substantial contribution to offset a short-fall in basic supplies. To this end the programmes of research and development in the fields of hatchery production, environmental control, nutritional innovation, genetic manipulation and disease identification and control within the species at present exploited or showing potential for exploitation require careful integration. The list of species at present includes rainbow trout, salmon and turbot with secondary consideration for brown trout, sea trout and sole.
THE term "vitamin(e)" was coined by Casimir Funk in 1912. He was good at promoting its use, but he could never have foreseen the mind-expanding effect of this new word in the 1970s. Vitamins are coenzymes and other metabolic catalysts whose pathways of biochemical synthesis have been lost as a result of mutations during the evolution of animals. Identification and synthesis of vitamins led to spectacular successes in preventive medicine; these became well known, and, as one result, popping vitamin pills is part of the American way of life, just like colour television in the family room. The idea is not so much that diets are deficient, but why not take vitamins "just to be safe"? Indeed, except for a half-hearted attempt some years ago by the US Food and Drug Administration (FDA) to throw cold water on it, the habit had few opponents and it was certainly better than smoking cigarettes.

There is, however, a basic pharmacological delusion of the layman that if a small dose of a medicine is good, a large dose must be even better, especially if it is not poisonous. Many physicians have treated patients whose symptoms exceeded in severity their physical diagnosis, by prescribing "a good rest with lots of vitamins". This procedure is known as "placebo therapy". the administration of a is that such dosage will produce superharmless nostrum for soothing the health, an effect not measurable in nerves of the apprehensive, and for human beings or laboratory animals.
convincing those who are open to Vitamins go through periods of convincing those who are open to

\section{Funk therapy}

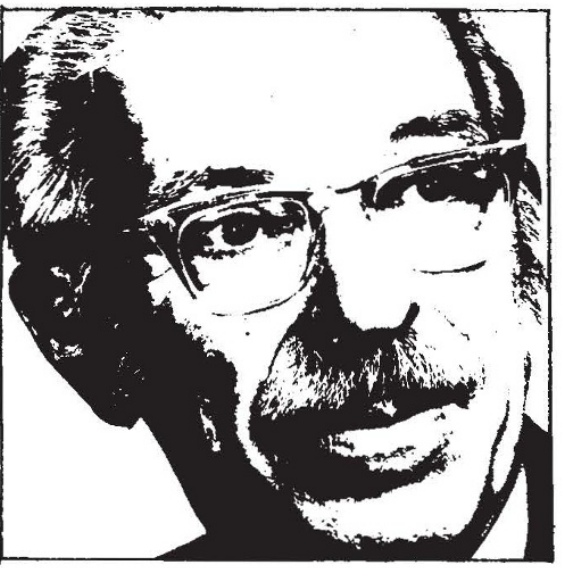

THOMAS H. JUKES

suggestion that they are receiving "medical treatment". Vitamins can be sold without prescription, and the medical profession, by fostering the use of vitamin pills, made an opening for nonphysicians to practice medicine without a licence. The "health-food" industry responded, and its sales outlets stock pills containing vitamins and alleged vitamins. If health food is so "nutritious", why does it need supplementation with large doses of vitamins and minerals? The answer given in the health food literature fashion. Thiamine was in the spotlight at one time and, more recently, ascorbic acid became the darling of the headlines. Vitamin $\mathbf{B}_{12}$ is popular, probably because it is often injected and is red. Vitamin $\mathbf{B}_{6}$ is all the rage now, and vitamin $\mathrm{E}$ is a hardy perennial because it was lucky enough to be discovered by the fact that its deficiency causes infertility in rats. The same is true of vitamin A, of course, but $\mathrm{E}$ has ever since worn the halo of Aphrodite, and has collected many unearned credits.

"Megavitamin therapy" has its devotees, and publicity is currently given to "orthomolecular psychiatry", defined as the "achievement and preservation of good mental health by the provision of the optimum molecular environment for the mind, especially the optimum concentrations of substances normally present in the human body, such as the vitamins". Whether or not this actually works we shall probably never know, because the author (Linus Pauling) also says "the principles of medical ethics prevent orthomolecular psychiatrists from withholding from half of their patients a treatment that they consider to be valuable. Controlled tests can be carried out only by skeptics".

Efforts of the FDA to regulate megavitamin promotion, however, were set back by a court decision and by the passage last year of a bill in the US Senate that specifically prevents the FDA from classifying highpotency vitamin preparations as drugs. 